Commun. Fac. Sci. Univ. Ank. Ser. A1 Math. Stat.

Volume 69, Number 1, Pages 307-319(2020)

DOI: $10.31801 /$ cfsuasmas. 456764

ISSN 1303-5991 E-ISSN 2618-6470

http://communications.science.ankara.edu.tr/index.php?series=A1

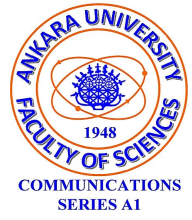

\title{
LIGHTLIKE HYPERSURFACES AND LIGHTLIKE FOCAL SETS WITH RESPECT TO BISHOP FRAME IN 4-DIMENSIONAL MINKOWSKI SPACE $\mathbb{E}_{1}^{4}$
}

\author{
ZEHRA OZDEMIR
}

\begin{abstract}
In this article, light-like hypersurfaces which are derived by null Cartan curves are examined and discussed. The singularities of lightlike hypersurfaces and light-like focal sets are investigated by using the Bishop frame on the Null Cartan curves. We obtain that the types of these singularities and the order of contact between the null Cartan curves are closely related to the Bishop curvatures of the null Cartan curves. Moreover, two examples of light-like hypersurfaces and light-like focal sets are given to illustrate our theoretical results.
\end{abstract}

\section{INTRODUCTION}

In 4-dimensional Minkowski space, due to the causal character there are three categories of vectors, namely, space-like, time-like and light-like (null) ones. Therefore, hypersurfaces of a Lorentzian manifold $(M, g)$ can be of three types(see [16]): Space-like, time-like and light-like (null) hypersurfaces. Especially, the geometry of light-like hypersurfaces becomes more difficult and is completely different from that of the space-like and time-like hypersurfaces. In the case of light-like (degenerate, null) hypersurfaces, the situation is totally different. The normal bundle $T M^{\perp}$ is a rank-one distribution on $M: T M^{\perp} \subset T M$. It is also coincides with the radical distribution $\operatorname{RadTM}=T M \cap T M^{\perp}$. Therefore, the induced metric $g$ is degenerate on $M$ and it has a constant rank $n$. Therefore, these hypersurfaces are usually used in modeling objects that are difficult to understand. In particular, light-like hypersurfaces are of interest to physicists because Kerr black holes, and various horizons can be modeled with these hypersurfaces [2, 4, 10, 11, 13, 17, 18, 20, 24. Moreover, these hypersurfaces are used in the electromagnetism theory [19, 21]. Nersessian and Ramos have shown that there is a geometric particle-model based on the geometry of null curves in Minkowski 4-space [14]. Moreover, they studied

Received by the editors: September 03, 2018; Accepted: October 15, 2019.

2010 Mathematics Subject Classification. 53C50, 53C40,57R45, 53B30, 53B50.

Key words and phrases. Bishop frame, null Cartan curve, lightlike hypersurface, singularity. 
the geometric particle model related to the null curves in Minkowski 3-space [15]. Duggal et.al. gave various fundamental works for the differential geometry theory of light-like submanifolds [5, 6, 7, 8,

On the other hand, the use of differential geometry in the singularity theory was first demonstrated by Thom in 1965. This study provides a connection between physics and geometry. Then, in 1998, Akivis et al. investigate the singular points of light-like hypersurfaces of the de Sitter space $S^{n+1}[1$. The singularities of light-like surface and hypersurfaces have been studied in [22, 23.

In this study, the singularities of the hypersurfaces are defined by using the Bishop frame of the null Cartan curve in Minkowski 4-space. We will classify singular points of light-like hypersurfaces and light-like focal sets. We have also shown that the types of these singularities are closely related to the curvatures of the Bishop Cartan curvatures. Finally, we visualized light-like hypersurfaces and light-like focal set to demonstrate our theoretical results.

\section{Preliminaries}

The 4-dimensional Minkowski space-time is a real vector space $\mathbb{E}_{1}^{4}$ furnished with a symmetric non-degenerate $(0,2)$ tensor field $g$ with constant index. The metric tensor $g$ on $\mathbb{E}_{1}^{4}$ with the signature $(-,+,+,+)$ has the form

$$
g(x, y)=-x_{1} y_{1}+x_{2} y_{2}+x_{3} y_{3}+x_{4} y_{4}
$$

for any two $x=\left(x_{1}, x_{2}, x_{3}, x_{4}\right)$ and $y=\left(y_{1}, y_{2}, y_{3}, y_{4}\right)$ in $\mathbb{E}_{1}^{4}$.

A non-zero vector $x$ of $\mathbb{E}_{1}^{4}$ is called space-like if $g(x, x)>0$, time-like if $g(x, x)<0$ and null if $g(x, x)=0$. Any two vectors $x, y \in \mathbb{E}_{1}^{4}$ are called orthogonal if $g(x, y)=$ 0 . Any two null vectors are called orthogonal on the condition that they are linearly dependent.

Let $\alpha: I \rightarrow \mathbb{E}_{1}^{4} ; w \rightarrow \alpha(w)$ be a smooth curve in $\mathbb{E}_{1}^{4}$. Then the tangent vector of the curve denoted by

$$
t=\frac{d \alpha}{d w} .
$$

The curve $\alpha$ is said to be a null (isotropic or light-like ) curve iff locally at each point it satisfies

$$
g\left(\frac{d \alpha}{d w}, \frac{d \alpha}{d w}\right)=0
$$

The null curve parameterized by the pseudo-arc parameter $s$ denoted by

$$
s(w)=\int_{0}^{w} g\left(\alpha^{\prime \prime}(w), \alpha^{\prime \prime}(w)\right) d w .
$$

is called as a null Cartan Curve. The Cartan frame $\left\{t, n, b_{1}, b_{2}\right\}$ along the nongeodesic null Cartan curve $\alpha$ satisfies the following Cartan frame equations

$$
\begin{aligned}
t^{\prime} & =k_{1} n \\
n^{\prime} & =-k_{2} t+k_{1} b_{1}
\end{aligned}
$$




$$
\begin{aligned}
b_{1}^{\prime} & =-k_{2} n+k_{3} b_{2} \\
b_{2}^{\prime} & =k_{3} t
\end{aligned}
$$

here $k_{1}(s), k_{2}(s)$ and $k_{3}(s)$ are Cartan curvature functions and the first Cartan curvature $k_{1}(s)=1$ in pseudo-arc parameter $s$. The null Cartan curve is called a null Cartan cubic on the condition that the second Cartan curvature satisfies $k_{2}(s)=0$. Moreover, we have the following relations

$$
\begin{aligned}
g(t, t) & =g\left(b_{1}, b_{1}\right)=0, g(n, n)=g\left(b_{2}, b_{2}\right)=1, \\
g(t, n) & =g\left(t, b_{2}\right)=g\left(n, b_{1}\right)=g\left(b_{1}, b_{2}\right)=0, g\left(t, b_{1}\right)=-1
\end{aligned}
$$

[9].

Definition 1. The Bishop frame $\left\{t_{1}, n_{1}, n_{2}, n_{3}\right\}$ of a null Cartan curve in $\mathbb{E}_{1}^{4}$ is positively oriented pseudo-orthonormal frame. It contains a tangential vector field $t_{1}$, two relatively parallel space-like normal vector fields $n_{1}$ and $n_{3}$, and a relatively parallel light-like transversal vector field $n_{2}$. These vectors have satisfy the following conditions

$$
\begin{aligned}
g\left(t_{1}, t_{1}\right) & =g\left(n_{2}, n_{2}\right)=0, g\left(n_{1}, n_{1}\right)=g\left(n_{3}, n_{3}\right)=1, \\
g\left(t_{1}, n_{1}\right) & =g\left(n_{1}, n_{2}\right)=g\left(n_{1}, n_{3}\right)=g\left(n_{2}, n_{3}\right)=0, g\left(t_{1}, n_{2}\right)=-1
\end{aligned}
$$

[12.

Theorem 2. Let $\alpha$ be a null Cartan curve in $\mathbb{E}_{1}^{4}$ with the Cartan curvatures $k_{1}(s)=$ $1, k_{2}(s)$ and $k_{3}(s)=0$. Then the Bishop frame $\left\{t_{1}, n_{1}, n_{2}, n_{3}\right\}$ and the Cartan frame $\left\{t, n, b_{1}, b_{2}\right\}$ of $\alpha$ have the following relation

$$
\left[\begin{array}{l}
t_{1} \\
n_{1} \\
n_{2} \\
n_{3}
\end{array}\right]=\left[\begin{array}{cccc}
1 & 0 & 0 & 0 \\
-\sigma_{2} & 1 & 0 & 0 \\
\frac{\sigma_{2}^{2}}{2} & -\sigma_{2} & 1 & 0 \\
0 & 0 & 0 & 1
\end{array}\right]\left[\begin{array}{c}
t \\
n \\
b_{1} \\
b_{2}
\end{array}\right]
$$

and the Bishop frame derivative formulas are given as

$$
\left[\begin{array}{l}
t_{1}^{\prime} \\
n_{1}^{\prime} \\
n_{2}^{\prime} \\
n_{3}^{\prime}
\end{array}\right]=\left[\begin{array}{cccc}
\sigma_{2} & \sigma_{1} & 0 & 0 \\
0 & 0 & \sigma_{1} & 0 \\
0 & 0 & -\sigma_{2} & 0 \\
0 & 0 & 0 & 0
\end{array}\right]\left[\begin{array}{c}
t_{1} \\
n_{1} \\
n_{2} \\
n_{3}
\end{array}\right] .
$$

where the first Bishop curvature $\sigma_{1}(s)=1$, the third Bishop curvature $\sigma_{3}(s)=0$ and the second Bishop curvature satisfies the following differential equation

$$
\sigma_{2}^{\prime}(s)=-\frac{1}{2} \sigma_{2}^{2}(s)-k_{2}(s)
$$

12. 
Particularly, the relation for cross products of the Bishop frame vector fields are

$$
\begin{aligned}
& t_{1} \times n_{1} \times n_{2}=-n_{3} ; t_{1} \times n_{1} \times n_{3}=-t_{1} ; \\
& t_{1} \times n_{3} \times n_{2}=n_{1} ; n_{1} \times n_{2} \times n_{3}=-n_{2}
\end{aligned}
$$

[12.

Theorem 3. Let $\alpha$ be a null Cartan curve in $\mathbb{E}_{1}^{4}$ with the Cartan curvatures $k_{1}(s)=$ $1, k_{2}(s)$ and $k_{3}(s) \neq 0$. Then the Bishop frame vectors $\left\{t_{1}, n_{1}, n_{2}, n_{3}\right\}$ and the Cartan frame vectors $\left\{t, n, b_{1}, b_{2}\right\}$ of $\alpha$ are given by the following relation

$$
\left[\begin{array}{c}
t_{1} \\
n_{1} \\
n_{2} \\
n_{3}
\end{array}\right]=\left[\begin{array}{cccc}
1 & 0 & 0 & 0 \\
-\sigma_{1} \sigma_{2}-\sigma_{3} \sqrt{\sigma_{1}^{\prime 2}+\sigma_{3}^{\prime 2}} & \sigma_{1} & 0 & \sigma_{3} \\
\frac{\sigma_{2}^{2}+\sigma_{1}^{\prime 2}+\sigma_{3}^{\prime 2}}{2} & -\sigma_{2} & 1 & -\sqrt{\sigma_{1}^{\prime 2}+\sigma_{3}^{\prime 2}} \\
\sigma_{2} \sigma_{3}-\sigma_{1} \sqrt{\sigma_{1}^{\prime 2}+\sigma_{3}^{\prime 2}} & -\sigma_{3} & 0 & \sigma_{1}
\end{array}\right]\left[\begin{array}{c}
t \\
n \\
b_{1} \\
b_{2}
\end{array}\right]
$$

where the Bishop frame derivative formulas are presented as

$$
\left[\begin{array}{c}
t_{1}^{\prime} \\
n_{1}^{\prime} \\
n_{2}^{\prime} \\
n_{3}^{\prime}
\end{array}\right]=\left[\begin{array}{cccc}
\sigma_{2} & \sigma_{1} & 0 & -\sigma_{3} \\
0 & 0 & \sigma_{1} & 0 \\
0 & 0 & -\sigma_{2} & 0 \\
0 & 0 & -\sigma_{3} & 0
\end{array}\right]\left[\begin{array}{c}
t_{1} \\
n_{1} \\
n_{2} \\
n_{3}
\end{array}\right]
$$

where the first Bishop curvature $\sigma_{1}(s)=\sin \phi(s)$, the second Bishop curvature satisfies the differential equation

$$
\sigma_{2}(s)=\frac{k_{3}(s)-\phi^{\prime \prime}(s)}{\phi^{\prime}(s)}, \phi^{\prime}(s) \neq 0
$$

the third Bishop curvature $\sigma_{3}(s)=\cos \phi(s)$ and the function $\phi(s)$ satisfies the differential equation

$$
2 \phi^{\prime}\left(\phi^{\prime \prime \prime}-k_{3}^{\prime}\right)+2 \phi^{\prime \prime}\left(k_{3}-\phi^{\prime \prime}\right)+\phi^{4}-\left(k_{3}-\phi^{\prime \prime}\right)^{2}-2 k_{2} \phi^{2}=0
$$

where $\phi(s) \notin\left\{\frac{\pi}{2}+k \pi, k \pi\right\}, k \in \mathbb{Z}[12$.

Definition 4. The map $\mathbb{D G}_{C}^{ \pm}(u, \theta)$ is called as de Sitter Gauss image of $C=\alpha(I)$ with respect to Bishop frame in $\mathbb{E}_{1}^{4}$ and defined as

$$
\mathbb{D G}_{C}=U \times \mathbb{R} \rightarrow \mathbb{S}_{1}^{3} ; \mathbb{D G}_{C}(s, \eta, \theta)=\eta t_{1}(s)+\cos \theta n_{1}(s)+\sin \theta n_{3}(s) .
$$

Definition 5. The light-like hypersurfaces along $C$ with respect to Bishop frame in $\mathbb{E}_{1}^{4}$ defined by

$$
\mathbb{L} \mathbb{H}_{C}: U \times \mathbb{R} \rightarrow \mathbb{E}_{1}^{4} ; \mathbb{L}_{\mathbb{H}_{\alpha}}(s, \eta, \theta)=\alpha(s)+\mathbb{D} \mathbb{G}_{C}(s, \eta, \theta) .
$$

In the following section we derive the light-like hypersurfaces along $C$ and investigate the singularities of the light-like hypersurfaces. 


\section{Lightlike Hypersurfaces and Singularities}

Let $\alpha: I \rightarrow \mathbb{E}_{1}^{4}$ be a null Cartan curve with the Bishop frame apparatus $\left\{t_{1}, n_{1}, n_{2}, n_{3}\right\}$. Then the light-like distance squared function is defined as

$$
d(p, \xi)=g(\xi-\alpha(s), \xi-\alpha(s))-1
$$

here $p=\alpha(s)$ for any fixed $\xi_{0} \in \mathbb{E}_{1}^{4}$, we have

$$
\zeta(p)=\zeta \xi_{0}(p)=d\left(p, \xi_{0}\right)
$$

If we take derivative of the last equation we get

$$
\zeta^{\prime}(p)=-2 g\left(t_{1}(s), \xi_{0}-\alpha(s)\right) .
$$

Then, we calculate the discriminant set of the light-like squared function $d$ as follows

$$
\mathbb{D}_{d}=\left\{\xi=\alpha(s)+\eta t_{1}(s)+\cos \theta n_{1}(s)+\sin \theta n_{3}(s): \theta \in[0,2 \pi), s \in I, \eta \in \mathbb{R}\right\} .
$$

It is called as image of the light-like hypersurface along $C$. The second derivative of the function $\zeta(p)$ calculated

$$
\begin{aligned}
\zeta^{\prime \prime}(p) & \left.=-2 g\left(T_{1}^{\prime}(s), \xi_{0}-\alpha(s)\right)\right) \\
& \left.=-2 g\left(\left(\sigma_{2}(s) t_{1}(s)+\sigma_{1}(s) n_{1}(s)-\sigma_{3}(s) n_{3}(s)\right), \cos \theta n_{1}(s)+\sin \theta n_{3}(s)\right)\right) \\
& =-2 \sigma_{1}(s) \cos \theta+2 \sigma_{3}(s) \sin \theta
\end{aligned}
$$

from the Bishop frame equation which give following two case

Case 1. $\sigma_{3}(s)=0$,

iff

$$
\zeta(p)=\zeta^{\prime}(p)=\zeta^{\prime \prime}(p)=0
$$

Case 2. $\sigma_{3}(s) \neq 0$,

$$
\cos \theta=0
$$

iff

$$
\zeta(p)=\zeta^{\prime}(p)=\zeta^{\prime \prime}(p)=0
$$

$$
\tan \theta=\frac{\sigma_{1}(s)}{\sigma_{3}(s)} .
$$

Using the Bishop curvature equation we obtain

$$
\theta=\phi(s)+k \pi, k \in \mathbb{Z} .
$$

Therefore, singular points of the light-like hypersurfaces are points satisfy

(i) If $\cos \theta=0$ then the singular point of the light-like hypersurface is $\xi_{0}=$ $\alpha\left(s_{0}\right)+\eta_{0} t_{1}(s) \mp n_{3}(s)$ for $\eta_{0} \in \mathbb{R}$. Then we obtain the light-like focal sets as

$$
\mathbb{L} \mathbb{F} \mathbb{S}_{C}^{ \pm}=\left\{\xi=\alpha(s)+\mathbb{D G}_{C}\left(s, \eta, \pi \pm \frac{\pi}{2}\right): s \in I, \eta \in \mathbb{R}\right\}
$$

(ii) If $\theta=\phi+k \pi, k \in \mathbb{Z}$ then the singular points of the light-like hypersurfaces are points $\xi_{0}=\alpha\left(s_{0}\right)+\eta_{0} t_{1}(s)+\cos \theta n_{1}(s)+\sin \theta n_{3}(s)$.

Then we obtain the light-like focal sets as

$$
\left\{\mathbb{L} \mathbb{F S}_{C}=\xi=\alpha(s)+\mathbb{D G}_{C}(s, \eta, \phi(s)+k \pi,): k \in \mathbb{Z}, s \in I, \eta \in \mathbb{R}\right\}
$$


Using the above characterizations we obtain the following proposition.

Proposition 6. Let $\alpha$ be a curve null Cartan curve with the Bishop frame $\left\{t_{1}, n_{1}, n_{2}, n_{3}\right\}$. Then we have following three condition

1. $h(p)=\zeta^{\prime}(p)=0$ iff there exist $\theta_{0} \in[0,2 \pi)$ and $\eta_{0} \in \mathbb{R}$ such that

$$
\xi_{0}-p_{0}=\eta t_{1}\left(s_{0}\right)+\cos \theta n_{1}\left(s_{0}\right)+\sin \theta n_{3}\left(s_{0}\right) .
$$

2. $i$. If $\sigma_{3}(s)=0$, then we have,

$\zeta(p)=\zeta^{\prime}(p)=\zeta^{\prime \prime}(p)=0$ iff there exist $\theta_{0}=\pi \pm \frac{\pi}{2}$ and $\eta_{0} \in \mathbb{R}$ such that

$$
\xi_{0}-p_{0}=\eta_{0} t_{1}\left(s_{0}\right) \pm n_{3}\left(s_{0}\right) .
$$

ii. If $\sigma_{3}(s) \neq 0$, then we have,

$\zeta(p)=\zeta^{\prime}(p)=\zeta^{\prime \prime}(p)=0$ iff there exist $\theta_{0}=\phi(s)+k \pi, k \in \mathbb{Z}$ and $\eta_{0} \in \mathbb{R}$ such that

$$
\xi_{0}-p_{0}=\eta t_{1}(s)+\cos (\phi(s)+k \pi) n_{1}(s)+\sin (\phi(s)+k \pi) n_{3}(s) .
$$

3.i. If $\sigma_{3}(s)=0$, then we have,

$\zeta(p)=\zeta^{\prime}(p)=\zeta^{\prime \prime}(p)=\zeta^{\prime \prime \prime}(p)=0$ iff there exist $\theta_{0}=\pi \pm \frac{\pi}{2}$ and $\eta_{0}=0$ such that

$$
\xi_{0}-p_{0}= \pm n_{3}\left(s_{0}\right) \text {. }
$$

ii. If $\sigma_{3}(s) \neq 0$, then we have,

$\zeta(p)=\zeta^{\prime}(p)=\zeta^{\prime \prime}(p)=\zeta^{\prime \prime \prime}(p)=0$ iff there exist $\theta_{0}=\phi_{0}=\frac{\pi}{4}+2 k \pi, k \in \mathbb{Z}$ and $\eta_{0}=0$ such that

$$
\xi_{0}-p_{0}=\eta t_{1}(s)+\cos \left(\frac{\pi}{4}+2 k \pi\right) n_{1}(s)+\sin \left(\frac{\pi}{4}+2 k \pi\right) n_{3}(s) .
$$

4. If $\zeta(p)=\zeta^{\prime}(p)=\zeta^{\prime \prime}(p)=\zeta^{\prime \prime \prime}(p)$ then we have $\zeta^{(4)}(p) \neq 0$.

Definition 7. Let $\alpha: I \rightarrow \mathbb{E}_{1}^{4}$ be a null Bishop Cartan curve in $\mathbb{E}_{1}^{4}$. Then, the pseudo-sphere that have five-point contact with $\alpha$ is said to be the osculating pseudosphere of $\alpha[3]$.

Corollary 8. Let $\alpha: I \rightarrow \mathbb{E}_{1}^{4}$ be a null Bishop Cartan curve in $\mathbb{E}_{1}^{4}$. Then the curve $\alpha$ has not fife-point contact with osculating pseudo-sphere.

Proposition 9. If $\zeta_{\xi_{0}}\left(s_{0}\right)$ has $A_{k}$-singularity at $s_{0}(k=1,2,3,4)$ then it is a versal unfolding of $\zeta_{\xi_{0}}\left(s_{0}\right)$.

Proof. Let we give

$$
\alpha(s)=\left(x_{1}(s), x_{2}(s), x_{3}(s), x_{4}(s)\right), \xi(s)=\left(\xi_{1}, \xi_{2}, \xi_{3}, \xi_{4}\right)
$$

in $\mathbb{E}_{1}^{4}$. Then we know that if $h(s)$ has $A_{1}$ singularity at $s_{0}$ then we have

$$
\delta_{1}=\left(-2\left(\xi_{1}-x_{1}\left(s_{0}\right)\right),-2\left(\xi_{2}-x_{2}\left(s_{0}\right)\right),-2\left(\xi_{3}-x_{3}\left(s_{0}\right)\right),-2\left(\xi_{4}-x_{4}\left(s_{0}\right)\right)\right)
$$


Since $\xi-\alpha(s) \in \mathbb{S}_{1, \xi}^{3}$ we calculate that $\operatorname{rank} \delta_{1}=1$

Let we assume that $h_{\xi_{0}}\left(s_{0}\right)$ has $A_{k}$-singularity at $s_{0}(k=2,3,4)$ then we have the following matrix form

$$
\delta_{2}=\left[\begin{array}{cccc}
\left(\xi_{1}-x_{1}\left(s_{0}\right)\right. & \xi_{2}-x_{2}\left(s_{0}\right) & \xi_{3}-x_{3}\left(s_{0}\right) & \xi_{4}-x_{4}\left(s_{0}\right) \\
x_{1}^{\prime}\left(s_{0}\right) & x_{2}^{\prime}\left(s_{0}\right) & x_{3}^{\prime}\left(s_{0}\right) & x_{4}^{\prime}\left(s_{0}\right) \\
x_{1}^{\prime \prime}\left(s_{0}\right) & x_{2}^{\prime \prime}\left(s_{0}\right) & x_{3}^{\prime \prime}\left(s_{0}\right) & x_{4}^{\prime \prime}\left(s_{0}\right) \\
x_{1}^{\prime \prime \prime}\left(s_{0}\right) & x_{2}^{\prime \prime \prime}\left(s_{0}\right) & x_{3}^{\prime \prime \prime}\left(s_{0}\right) & x_{4}^{\prime \prime \prime}\left(s_{0}\right)
\end{array}\right]
$$

this give following two condition

$i$. If $\sigma(3) \neq 0$ then we have

$$
\xi-\alpha(s)=\eta t_{1}(s)+\cos (\phi(s)+k \pi) n_{1}(s)+\sin (\phi(s)+2 \pi) n_{3}(s) .
$$

The determinant of the matrix $\delta_{2}$ calculated as

$$
\begin{aligned}
\operatorname{det} \delta_{2}= & g\left((\xi-\alpha(s)) \times \alpha^{\prime}(s) \times \alpha^{\prime \prime}(s), \alpha^{\prime \prime \prime}(s)\right) \\
= & g\left(\eta t_{1}(s)+\cos (\phi(s)+k \pi) n_{1}(s)\right. \\
& \left.+\sin (\phi(s)+k \pi) n_{3}(s)\right) \times t_{1}(s) \times\left(\sigma_{2}(s) t_{1}(s)+\sigma_{1}(s) n_{1}(s)-\sigma_{3}(s) n_{3}(s)\right) \\
& ,\left(\sigma_{2}^{\prime}(s)+\sigma_{2}^{2}(s)\right) t_{1}(s)+\left(\sigma_{1}^{\prime}(s)+\sigma_{1}(s) \sigma_{2}(s)\right) n_{1}(s) \\
& \left.+\left(\sigma_{1}^{2}(s)+\sigma_{3}^{2}(s)\right) n_{2}(s)-\left(\sigma_{3}^{\prime}(s)+\sigma_{3}(s) \sigma_{2}(s)\right) n_{3}(s)\right) \\
= & g\left(-\sigma_{3} \cos (\phi(s)+k \pi) n_{1}(s) \times T_{1}(s) \times n_{3}(s)\right. \\
& +\sigma_{1} \sin (\phi(s)+k \pi) n_{3}(s) \times t_{1}(s) \times n_{1}(s) \\
& ,\left(\sigma_{2}^{\prime}(s)+\sigma_{2}^{2}(s)\right) t_{1}(s)+\left(\sigma_{1}^{\prime}(s)+\sigma_{1}(s) \sigma_{2}(s)\right) n_{1}(s) \\
& \left.+\left(\sigma_{1}^{2}(s)+\sigma_{3}^{2}(s)\right) n_{2}(s)-\left(\sigma_{3}^{\prime}(s)+\sigma_{3}(s) \sigma_{2}(s)\right) n_{3}(s)\right) \\
& g\left(-\sigma_{3} \cos (\phi(s)+k \pi) t_{1}(s)-\sigma_{1} \sin (\phi(s)+k \pi) t_{1}(s)\right. \\
& ,\left(\sigma_{2}^{\prime}(s)+\sigma_{2}^{2}(s)\right) t_{1}(s)+\left(\sigma_{1}^{\prime}(s)+\sigma_{1}(s) \sigma_{2}(s)\right) n_{1}(s) \\
& \left.+\left(\sigma_{1}^{2}(s)+\sigma_{3}^{2}(s)\right) n_{2}(s)-\left(\sigma_{3}^{\prime}(s)+\sigma_{3}(s) \sigma_{2}(s)\right) n_{3}(s)\right)
\end{aligned}
$$

from the Bishop curvature equation we calculated as

$$
\operatorname{det} \delta_{2}=\cos (k \pi)= \pm 1 \text {. }
$$

ii. If $\sigma(3)=0$ then we have

$$
\xi-\alpha(s)=\eta t_{1}(s) \pm n_{3}(s) .
$$

The determinant of the matrix $\delta_{2}$ calculated as

$$
\begin{aligned}
\operatorname{det} \delta_{2}= & g\left((\xi-\alpha(s)) \times \alpha^{\prime}(s) \times \alpha^{\prime \prime}(s), \alpha^{\prime \prime \prime}(s)\right) \\
= & g\left(\left(\eta t_{1}(s) \pm n_{3}(s)\right) \times t_{1} \times\left(\sigma_{2} t_{1}+\sigma_{1} N_{1}\right)\right. \\
& \left.,\left(\sigma_{2}^{\prime}+\sigma_{2}^{2}\right) t_{1}+\left(\sigma_{1}^{\prime}+\sigma_{1} \sigma_{2}\right) n_{1}+\sigma_{1}^{2} n_{2}\right)
\end{aligned}
$$

from the Bishop curvature equation we calculated as

$$
\operatorname{det} \delta_{2}= \pm 1
$$


This gives $\operatorname{det} \delta_{2} \neq 0$. These complete the proof.

Now we may give the following main theorem.

Let $\alpha: I \rightarrow \mathbb{E}_{1}^{4}$ be a null Cartan curve with the Cartan Bishop frame $\left\{t_{1}, n_{1}, n_{2}, n_{3}\right\}$ in $\mathbb{E}_{1}^{4}$. Then the lightlike hypersurfaces $\mathbb{L} \mathbb{H} \mathbb{C}(s, \mu, \theta)=\alpha(s)+\mu t(s)+\cos \theta n_{1}(s)+$ $\sin \theta n_{3}(s)$, satisfy the following assertions:

1.The pseudosphere $\mathbb{S}_{1, \xi_{0}}^{3}$ and the null Bishop Cartan curve $\alpha$ have at least a twopoint contact.

2. $i$. The pseudosphere $\mathbb{S}_{1, \xi_{0}}^{3}$ and the null Bishop Cartan curve $\alpha$ have a three-point contact iff there exist $\theta_{0}=\pi \pm \frac{\pi}{2}$ and $\eta_{0} \in \mathbb{R}$ such that

$$
\xi_{0}-p_{0}=\eta_{0} t_{1}\left(s_{0}\right) \pm n_{3}\left(s_{0}\right) .
$$

where the third Bishop curvature $\sigma_{3}(s)=0$.

ii. the pseudosphere $\mathbb{S}_{1, \xi_{0}}^{3}$ and the null Bishop Cartan curve $\alpha$ have a three-point contact iff there exist $\theta_{0}=\phi(s)+k \pi, k \in \mathbb{Z}$ and $\eta_{0} \in \mathbb{R}$ such that

$$
\xi_{0}-p_{0}=\eta t_{1}(s)+\cos (\phi(s)+k \pi) n_{1}(s)+\sin (\phi(s)+k \pi) n_{3}(s)
$$

where the third Bishop curvature $\sigma_{3}(s) \neq 0$.

Remark 1.Under this condition the light-like focal set $\mathbb{L} \mathbb{F} \mathbb{S}_{C}^{ \pm}$is non-singular. In addition, the light-like hypersurfaces is locally diffeomorphic to $C(2,3) \times \mathbb{R}^{2}$ at $\xi_{0}$. 3. $i$. The pseudosphere $\mathbb{S}_{1, \xi_{0}}^{3}$ and the null Bishop Cartan curve $\alpha$ have a four-point contact on the condition that there exist $\theta_{0}=\pi \pm \frac{\pi}{2}$ and $\eta_{0}=0$ such that

$$
\xi_{0}-p_{0}= \pm n_{3}\left(s_{0}\right) .
$$

where the third Bishop curvature $\sigma_{3}(s)=0$.

ii. The pseudosphere $\mathbb{S}_{1, \xi_{0}}^{3}$ and the null Bishop Cartan curve $\alpha$ have a four-point contact on the condition that there exist $\theta_{0}=\phi_{0}=\frac{\pi}{4}+2 k \pi, k \in \mathbb{Z}$ and $\eta_{0}=0$ such that

$$
\xi_{0}-p_{0}=\eta t_{1}(s)+\cos \left(\frac{\pi}{4}+2 k \pi\right) n_{1}(s)+\sin \left(\frac{\pi}{4}+2 k \pi\right) n_{3}(s)
$$

where the third Bishop curvature $\sigma_{3}(s) \neq 0$.

Remark 2.Under this condition the critical value set of the $\mathbb{L} \mathbb{F} \mathbb{S}_{C}$ is a regular curve and the focal set $\mathbb{L} \mathbb{F} \mathbb{S}_{C}$ is locally diffeomorphic to $C(2,3,4) \times \mathbb{R}^{2}$. Also, the light-like hypersurfaces is locally diffeomorphic to $S W \times \mathbb{R}$ at $\xi_{0}$.

4. The null Bishop Cartan curve $\alpha$ and pseudosphere $\mathbb{S}_{1, \xi_{0}}^{3}$ not have five-point contact.

In this section we provide two examples in $\mathbb{E}_{1}^{4}$. The first example is given for the case of $\sigma_{3}(s) \neq 0$ and the second example is for the case of $\sigma_{3}(s)=0$, to verify the given theory. 


\section{Applications}

Example 10. Let $\alpha$ be a following parameterized curve in $\mathbb{E}_{1}^{4}$

$$
\alpha(s)=\left(\sinh \frac{s}{\sqrt{2}}, \frac{1}{\sqrt{3}} \sin \frac{\sqrt{3} s}{\sqrt{2}}, \cosh \frac{s}{\sqrt{2}},-\frac{1}{\sqrt{3}} \cos \frac{\sqrt{3} s}{\sqrt{2}}\right)
$$

[12. Then the parallel transport frame of the curve $\alpha$ calculated as follows:

$$
\begin{aligned}
& t_{1}=\left(\frac{1}{\sqrt{2}} \cosh \frac{s}{\sqrt{2}}, \frac{1}{\sqrt{2}} \cos \frac{\sqrt{3} s}{\sqrt{2}}, \frac{1}{\sqrt{2}} \sinh \frac{s}{\sqrt{2}}, \frac{1}{\sqrt{2}} \sin \frac{\sqrt{3} s}{\sqrt{2}}\right) \\
& n_{1}=\left(\begin{array}{c}
\left(-\frac{1}{2} \sin \frac{\sqrt{3} s}{\sqrt{2}}-\frac{\sqrt{3}}{2} \cos \frac{\sqrt{3} s}{\sqrt{2}}\right) \cosh \frac{s}{\sqrt{2}}+\frac{1}{2} \sin \frac{\sqrt{3} s}{\sqrt{2}} \sinh \frac{s}{\sqrt{2}} \\
+\frac{3}{2 \sqrt{3}} \cos \frac{\sqrt{3} s}{\sqrt{2}} \sinh \frac{s}{\sqrt{2}}, \\
\left(-\frac{1}{2} \sin \frac{\sqrt{3} s}{\sqrt{2}}-\frac{\sqrt{3}}{2} \cos \frac{\sqrt{3} s}{\sqrt{2}}\right) \cos \frac{\sqrt{3} s}{\sqrt{2}}-\frac{\sqrt{3}}{2} \sin \frac{\sqrt{3} s}{\sqrt{2}} \sin \frac{\sqrt{3} s}{\sqrt{2}} \\
+\frac{1}{2} \cos \frac{\sqrt{3} s}{\sqrt{2}} \sin \frac{\sqrt{3} s}{\sqrt{2}}, \\
\left(-\frac{1}{2} \sin \frac{\sqrt{3} s}{\sqrt{2}}-\frac{\sqrt{3}}{2} \cos \frac{\sqrt{3} s}{\sqrt{2}}\right) \sinh \frac{s}{\sqrt{2}}+\frac{1}{\sqrt{2}} \sin \frac{\sqrt{3} s}{\sqrt{2}} \cosh \frac{s}{\sqrt{2}} \\
+\frac{3}{2 \sqrt{3}} \cos \frac{\sqrt{3} s}{\sqrt{2}} \cosh \frac{s}{\sqrt{2}}, \\
\left(-\frac{1}{2} \sin \frac{\sqrt{3} s}{\sqrt{2}}-\frac{\sqrt{3}}{2} \cos \frac{\sqrt{3} s}{\sqrt{2}}\right) \sin \frac{\sqrt{3} s}{\sqrt{2}}+\frac{\sqrt{3}}{2} \sin \frac{\sqrt{3} s}{\sqrt{2}} \cos \frac{\sqrt{3} s}{\sqrt{2}} \\
-\frac{1}{2} \cos \frac{\sqrt{3} s}{\sqrt{2}} \cos \frac{\sqrt{3} s}{\sqrt{2}}
\end{array}\right) \\
& n_{2}=\left(\sqrt{2} \cosh \frac{s}{\sqrt{2}}-\sqrt{2} \sinh \frac{s}{\sqrt{2}}, 0, \sqrt{2} \sinh \frac{s}{\sqrt{2}}-\sqrt{2} \cosh \frac{s}{\sqrt{2}}\right) \\
& n_{3}=\left(\begin{array}{c}
\left(\frac{1}{2} \cos \frac{\sqrt{3} s}{\sqrt{2}}-\frac{\sqrt{3}}{2} \sin \frac{\sqrt{3} s}{\sqrt{2}}\right) \cosh \frac{s}{\sqrt{2}}-\frac{1}{2} \cos \frac{\sqrt{3} s}{\sqrt{2}} \sinh \frac{s}{\sqrt{2}} \\
+\frac{3}{2 \sqrt{3}} \sin \frac{\sqrt{3} s}{\sqrt{2}} \sinh \frac{s}{\sqrt{2}}, \\
\left(\frac{1}{2} \cos \frac{\sqrt{3} s}{\sqrt{2}}-\frac{\sqrt{3}}{2} \sin \frac{\sqrt{3} s}{\sqrt{2}}\right) \cos \frac{\sqrt{3} s}{\sqrt{2}}+\frac{\sqrt{3}}{2} \cos \frac{\sqrt{3} s}{\sqrt{2}} \sin \frac{\sqrt{3} s}{\sqrt{2}} \\
+\frac{1}{2} \sin \frac{\sqrt{3} s}{\sqrt{2}} \sin \frac{\sqrt{3} s}{\sqrt{2}} \\
\left(\frac{1}{2} \cos \frac{\sqrt{3} s}{\sqrt{2}}-\frac{\sqrt{3}}{2} \sin \frac{\sqrt{3} s}{\sqrt{2}}\right) \sinh \frac{s}{\sqrt{2}}-\frac{1}{\sqrt{2}} \cos \frac{\sqrt{3} s}{\sqrt{2}} \cosh \frac{s}{\sqrt{2}} \\
+\frac{3}{2 \sqrt{3}} \sin \frac{\sqrt{3} s}{\sqrt{2}} \cosh \frac{s}{\sqrt{2}}, \\
\left(\frac{1}{2} \cos \frac{\sqrt{3} s}{\sqrt{2}}-\frac{\sqrt{3}}{2} \sin \frac{\sqrt{3} s}{\sqrt{2}}\right) \sin \frac{\sqrt{3} s}{\sqrt{2}}-\frac{\sqrt{3}}{2} \cos \frac{\sqrt{3} s}{\sqrt{2}} \cos \frac{\sqrt{3} s}{\sqrt{2}} \\
-\frac{1}{2} \sin \frac{\sqrt{3} s}{\sqrt{2}} \cos \frac{\sqrt{3} s}{\sqrt{2}}
\end{array}\right)
\end{aligned}
$$

We can give the light-like hypersurface as the form $\mathbb{L} \mathbb{H} \mathbb{C}(s, \mu, \theta)=\alpha(s)+\mu T_{1}(s)+$ $\cos \theta n_{1}(s)+\sin \theta n_{3}(s)$. When $\mu=0$, it is a ruled hypersurface derived by using the null parallel transport frame. We draw the projections of surface $\mathbb{L} \mathbb{H} \mathbb{C}(s, 0, \theta)$ on 3-dimensional space. Also, we plotted the projections of the critical value focal set $\mathbb{L} \mathbb{F} \mathbb{C}\left(s, 0, \frac{\pi}{4}\right)$ on 3 dimensional space illustrated in Figure 2. Thus, we may provide the clue for the image of the light-like hypersurface $\mathbb{L} \mathbb{H} \mathbb{C}(s, 0, \theta)$ with view of the null parallel transport frame via these projections.

Remark 2. The yellow-orange parts correspond to the Focal set $\mathbb{L} \mathbb{F} \mathbb{S}_{C}\left(s, \eta, \frac{\pi}{4}\right)$, $s \in I, \eta \in \mathbb{R}$, the red parts correspond to the critical value set $\mathbb{L} \mathbb{F} \mathbb{C}\left(s, 0, \frac{\pi}{4}\right)$. 

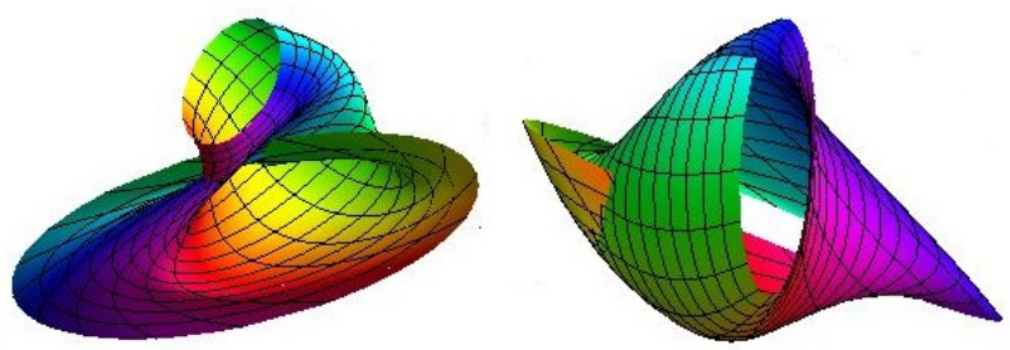

FiguRE 1. Projections of surface $\mathbb{L} \mathbb{H} \mathbb{C}(s, 0, \theta)$ on 3-dimensional space.

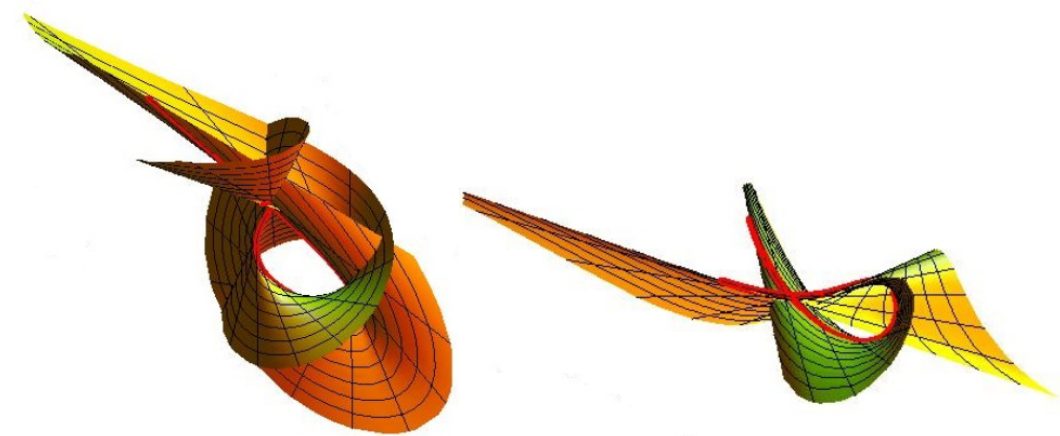

Figure 2. Projections of critical value set $\mathbb{L F} \mathbb{C}\left(s, 0, \frac{\pi}{4}\right)$ on 3dimensional space.

Example 11. Let $\alpha$ be a following parameterized curve in $\mathbb{E}_{1}^{4}$

$$
\alpha(s)=\left(\begin{array}{c}
\frac{1}{6} s^{3}+\frac{1}{2} s \\
\frac{1}{6} s^{3}-\frac{1}{2} s \\
\frac{-\frac{1}{5} s^{2}+\frac{4}{5} s^{2} \tan \left(\frac{1}{2} \ln s\right)+\frac{1}{5} \tan ^{2}\left(\frac{1}{2} \ln s\right)}{1+\tan ^{2}\left(\frac{1}{2} \ln s\right)} \\
\frac{\frac{2}{5} s^{2}+\frac{2}{5} s^{2} \tan \left(\frac{1}{2} \ln s\right)-\frac{2}{5} \tan ^{2}\left(\frac{1}{2} \ln s\right)}{1+\tan ^{2}\left(\frac{1}{2} \ln s\right)}
\end{array}\right)
$$

23. Then the parallel transport frame of the curve $\alpha$ calculated as follows:

$$
t=\frac{\sqrt{2}}{2}\left(\frac{1}{2} s^{2}+\frac{1}{2}, \frac{1}{2} s^{2}-\frac{1}{2}, s \sin (\ln s), s \cos (\ln s)\right)
$$




$$
\begin{aligned}
& n_{1}=\left(\begin{array}{c}
\left(\frac{\sqrt{6} s^{\sqrt{3}-1}}{c+s^{\sqrt{3}}}\right)\left(\frac{1}{2} s^{2}+\frac{1}{2}\right)+\frac{\sqrt{2}}{2} s, \\
\left(\frac{\sqrt{6} s^{\sqrt{3}}-1}{c+s^{\sqrt{3}}}\right)\left(\frac{1}{2} s^{2}-\frac{1}{2}\right)+\frac{\sqrt{2}}{2} s, \\
\left(\frac{\sqrt{6} s^{\sqrt{3}-1}}{c+s^{\sqrt{3}}}\right) s \sin (\ln s)+\frac{\sqrt{2}}{2}(\cos (\ln s)+\sin (\ln s)), \\
\left(\frac{\sqrt{6} s^{\sqrt{3}}-1}{c+s^{\sqrt{3}}}\right) s \cos (\ln s)+\frac{\sqrt{2}}{2}(\cos (\ln s)-\sin (\ln s)
\end{array}\right) \\
& n_{2}=\left(\begin{array}{c}
\left(\frac{\sqrt{6} s^{\sqrt{3}-1}}{c+s^{\sqrt{3}}}\right)^{2}\left(\frac{1}{4} s^{2}+\frac{1}{4}\right)-\left(\frac{\sqrt{6} s^{\sqrt{3}-1}}{c+s^{\sqrt{3}}}\right) \frac{\sqrt{2}}{2} s-\frac{5 \sqrt{2}}{8}-\frac{\sqrt{2}}{8 s^{2}}, \\
\left(\frac{\sqrt{6} s^{\sqrt{3}}-1}{c+s^{\sqrt{3}}}\right)^{2}\left(\frac{1}{4} s^{2}-\frac{1}{4}\right)-\left(\frac{\sqrt{6} s^{\sqrt{3}}-1}{c+s^{\sqrt{3}}}\right) \frac{\sqrt{2}}{2} s-\frac{5 \sqrt{2}}{8}+\frac{\sqrt{2}}{8 s^{2}}, \\
\left(\frac{\sqrt{6} s^{\sqrt{3}-1}}{c+s^{\sqrt{3}}}\right)^{2} \frac{s \sin (\ln s)}{2}-\left(\frac{\sqrt{6} s^{\sqrt{3}-1}}{c+s^{\sqrt{3}}}\right) \frac{\sqrt{2}}{2}(\cos (\ln s)+\sin (\ln (s))) \\
-\frac{\sqrt{2}}{4 s}(2 \cos (\ln s)-\sin (\ln s)), \\
\left(\frac{\sqrt{6} s^{\sqrt{3}-1}}{c+s^{\sqrt{3}}}\right)^{2} \frac{s \cos (\ln s)}{2}-\left(\frac{\sqrt{6} s^{\sqrt{3}-1}}{c+s^{\sqrt{3}}}\right) \frac{\sqrt{2}}{2}(\cos (\ln s)-\sin (\ln (s)) \\
+\frac{\sqrt{2}}{4 s}(2 \sin (\ln s)+\cos (\ln s))
\end{array}\right) \\
& n_{3}=\frac{\sqrt{2}}{4}\left(s-\frac{1}{s}, s+\frac{1}{s},-2 \cos (\ln s)\right), 2 \sin (\ln s)
\end{aligned}
$$

We can give the light-like hypersurface as the form $\mathbb{L} \mathbb{H} \mathbb{C}(s, \mu, \theta)=\alpha(s)+\mu T_{1}(s)+$ $\cos \theta N_{1}(s)+\sin \theta N_{3}(s)$, when $\mu=0$, is a ruled hypersurface generated by using the null parallel transport frame. We draw the projections of surface $\mathbb{L} \mathbb{H} \mathbb{C}(s, 0, \theta)$ on 3-dimensional space. Also, we illustrated the projections of the critical value Focal set $\mathbb{L} \mathbb{F} \mathbb{C}^{\div}\left(s, 0, \pi \pm \frac{\pi}{2}\right)$ on 3 dimensional space illustrated in Figure 3 and Figure 4 . Thus, we can obtain the information for the image of the hypersurface $\mathbb{L} \mathbb{H} \mathbb{C}(s, 0, \theta)$ with view of the null parallel transport frame via these projections.

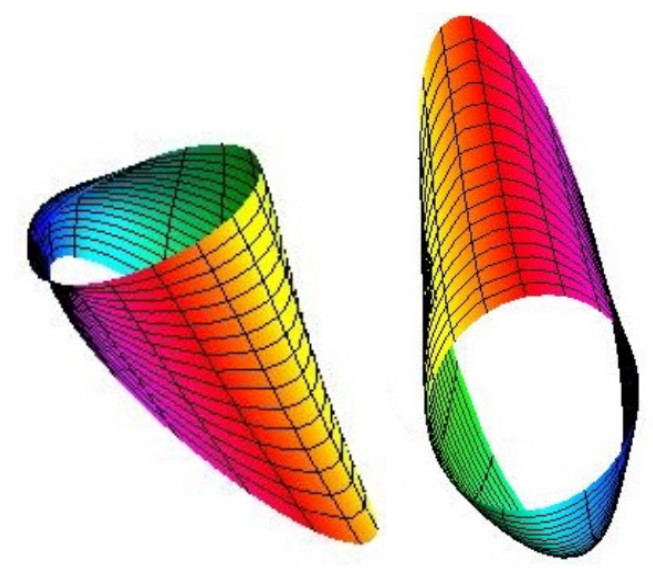

Figure 3. Projections of the hypersurface $\mathbb{L} \mathbb{H} \mathbb{C}(s, 0, \theta)$ on 3 dimensional space. 

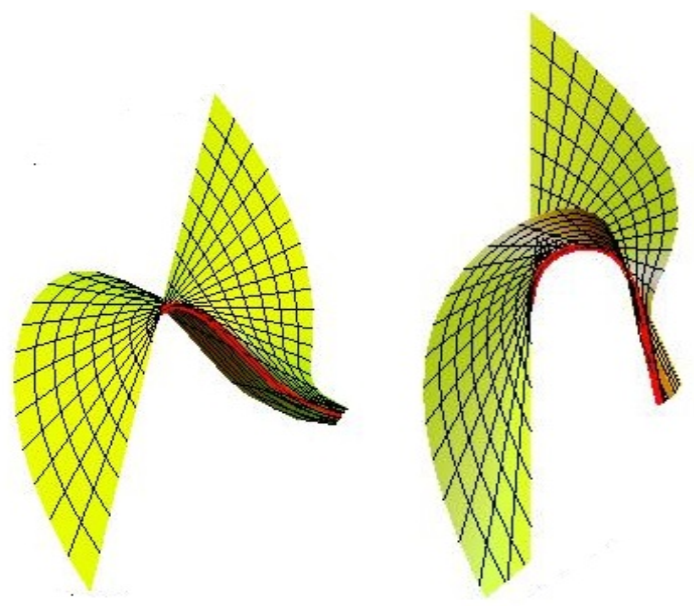

Figure 4. Projections of focal Set $\mathbb{L F} \mathbb{S}_{C}^{+}\left(s, \eta, \pi+\frac{\pi}{2}\right)$ on 3 dimensional space.
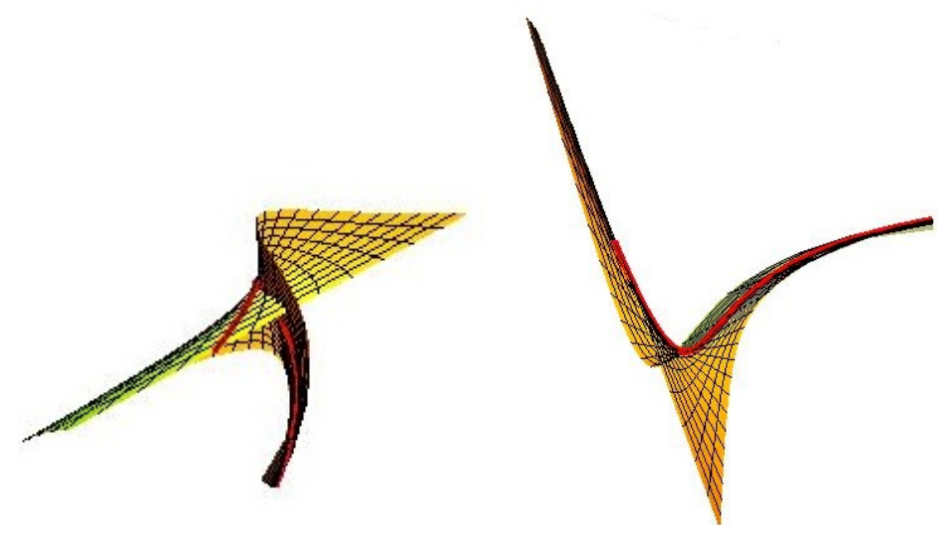

Figure 5. Projection of $\mathbb{L F} \mathbb{S}_{C}^{-}\left(s, \eta, \pi-\frac{\pi}{2}\right)$ on 3-dimensional space.

Remark 4. The yellow-orange parts correspond to the focal set $\mathbb{L} \mathbb{F} \mathbb{S}_{C}^{ \pm}\left(s, \eta, \pi \pm \frac{\pi}{2}\right)$, $s \in I, \eta \in \mathbb{R}$, the red parts correspond to the critical value focal set $\mathbb{L} \mathbb{F} \mathbb{S}_{C}^{ \pm}(s, \eta, \pi \pm$ $\left.\frac{\pi}{2}\right)$.

\section{REFERENCES}

[1] Akivis, M.A. and Goldberg, V.V., Singular points of lightlike hypersurfaces of the de Sitter space, Publications De L'Instıtut Mathematique, Nouvelle serie, 63(77), (1998), 81-101. 
[2] Bardeen, J. M. Carter, B. Hawking, S. W., The four laws of black hole mechanics, Comm. Math. Phys., 31, (1973), 161-170.

[3] Coken, A.C, CiftCi, U., On the Cartan curvatures of a null curve in Minkowski spacetime, Geometriae Dedicata, 114, (2005), 71-78.

[4] Clement, G., Black holes with a null Killing vector in three-dimensional massive gravity, Class. Quantum Grav., 26, (2009), 165002.

[5] Duggal, K.L. and Bejancu, A., Lightlike Submanifolds of Semi-Riemannian Manifolds and Applications, 364, Mathematics and its Aplications. Kluwer Academic Publishers Group, Dordrecht, The Netherlands, 1996.

[6] Duggal, K.L., Constant curvature and warped product globally null manifolds, J. Geom. Phy., 43, (2002), 327-340.

[7] Duggal, K.L., On scalar curvature in lightlike geometry, J. Geom. Phy., 57, (2007), 473-481.

[8] Duggal, K.L., A Report on canonical null curves and screen distributions for lightlike geometry, Acta Appl Math., 95, (2007), 135-149.

[9] Duggal, K.L. and Jin, D.H., Null Curves and Hypersurfaces of Semi-Riemannian Manifolds, World Scientific, Singapore, 2007.

[10] Gourgoulhon, E. and Jaramillo, J. L., A $3+1$ perspective on null hypersurfaces and isolated horizons, Phys. Rep. 423, (2006), 159-294.

[11] Hawking, S.W., Black holes in general relativity, Comm. Math. Phys., 25, (1972), 152-166.

[12] Ilarslan K. and Nešović, E., On Bishop frame of a null Cartan curve in Minkowski space-time, International Journal of Geometric Methods in Modern Physics, 15(8), (2018).

[13] Korzynski, M., Lewandowski,J. and Pawlowski, T., Mechanics of multidimensional isolated horizons, Class. Quantum Grav., 22, (2005), 2001-2016.

[14] Nersessian, A. and Ramos, E., Massive spinning particles and the geometry of null curves, Phys. Lett. B, 445, (1998), 123-128.

[15] Nersessian, A. and Ramos, E., A geometrical particle model for anyons, Modern Phys. Lett. A, 14, (1999), 2033-2037.

[16] O'Neill, B. Semi-Riemannian geometry, Academic Press, New York, 1983.

[17] Rudnicki, W., Black hole interiors cannot be totally vicious, Phys. Lett. A, 208, (1995), 53-58.

[18] Rudnicki, W., Budzynski, R. J. and Kondracki W., Generalized strong curvature singularities and weak cosmic censorship in cosmological space- times, Mod. Phys. Lett. A, 21, (2006), 1501-1509.

[19] de Souza, M.M., The Lorentz-Dirac equation and the structures of spacetime, Braz. J. Phys., $28,(1998), 250-256$.

[20] Sultana, J. and Dyer, C.C., Cosmological black holes: A black hole in the Einstein-de Sitter universe, Gen. Relativ. Gravit., 37, (2005), 1347-1370.

[21] Tertychniy, S.I., The black hole formed by electromagnetic radiation, Phys. Lett. A, 96, (1983), 73-75.

[22] Wang, Z. and Pei, D., Singularities of ruled null surfaces of the principal normal indicatrix to a null Cartan curve in de Sitter 3-space, Phys. Lett. B, 689, (2010), 101-106.

[23] Liu, X. and Wang, Z., On lightlike hypersurfaces and lightlike focal sets of null Cartan curves in Lorentz-Minkowski spacetime, J. Nonlinear Sci. Appl., (2015), 1-12.

[24] Vincent, M. and James, I., Symmetries of cosmological Cauchy horizons, Comm. Math. Phys., $89,(1983), 387-413$.

Current address: Zehra ÖZDEMİR: Department of Mathematics, Faculty of Science and Arts, Amasya University, Amasya, TURKEY

E-mail address: zehra.ozdemir@amasya.edu.tr

ORCID Address: http://orcid.org/0000-0001-9750-507X 